\title{
Two-electron temperature model of a laser-driven implosion
}

\author{
J. R. Sanmartin, R. Ramis, J. L. Montanes, and J. Sanz \\ Escuela Técnica Superior de Ingenieros Aeronáuticos, Universidad Politécnica de Madrid, Madrid-3, Spain
}

\begin{abstract}
The plasma ejected by a pellet irradiated with moderately intense laser light $\left(I_{\mathrm{cr}} \lambda^{2} \sim 10^{15} \mathrm{~W} \mathrm{~cm}^{-2}\right.$ $\mu \mathrm{m}^{2}, I_{\mathrm{cr}}$ and $\lambda$ being intensity and wavelength) is analyzed. Both hot electrons caused by resonant absorption, and cold or thermal electrons are considered; no appeal is made to heat-flux saturation. The cold (hot) population controis the overdense (underdense) plasma flow. Ingoing hot electrons at the critical surface are found to thermalize before reaching the ablation surface. Results obtained are compared with a model that assumes one electron temperature, and a saturated flux; agreement requires using a range of saturation factors: $0.1 \leqslant f \leqslant 0.3$. The validity of the model is discussed.
\end{abstract}

\section{INTRODUCTION}

A quasisteady fluid model was used in the past to analyze the coronal plasma ejected by a laser-irradiated pellet. ${ }^{1-4}$ The quasisteady assumption, discussed by Max et al., ${ }^{3}$ holds if a fluid particle crosses the corona in a time, of order $r_{\text {cr }} / v\left(r_{c r}\right)$, which is small compared with both $r_{\alpha} / \dot{r}_{a}$ and $W / \dot{W} ; r_{c r}$ is the radius at the critical density $\rho_{c r}, r_{a}(t)$ is the target or ablation radius, and $W(t)$ is the absorbed power. Using momentum conservation the first condition reads $r_{\mathrm{cr}} / r_{a}<u\left(r_{\mathrm{cr}}\right) / \dot{r}_{a} \sim\left|\rho\left(r_{a}\right) / \rho_{\mathrm{cr}}\right|^{1 / 2}$ and is usually satisfied: compression may increase $\rho\left(r_{a}\right)$ well above solid density, which, for $1.06 \mu \mathrm{m}$ light and an Al target, say, is about 770 $\rho_{\text {or }}$. Note that if the pulse lasts throughout the entire collapse we have $W / \dot{W} \sim r_{a} / \dot{r}_{a}$.

For $\rho\left(r_{a}\right) / \rho_{\mathrm{cr}} \rightarrow \infty$ the fluid model is quite simple. Because of the nonlinear character of heat conductivity, ${ }^{5}$ $K=\bar{K} T^{5 / 2}$, a sharp ablation front at $r_{a}$ uncouples the pellet from the corona. The corona is then parametrized by a dimensionless number, ${ }^{2,4} \hat{W}\left(W, r_{a}, n_{c r}, Z_{i}, \bar{m}\right) \equiv W / r_{a}^{2} \rho_{c r} V^{3}$, where $\rho_{\mathrm{cr}}$ is $\bar{m} n_{c r}, Z_{i}$ is the ion charge number, $\bar{m}=m_{i} / Z_{i}$ is the mass per unit charge, and $V$ is a convenient speed, ${ }^{6}$

$$
\begin{aligned}
V \equiv & \left(\frac{r_{a} n_{\mathrm{cr}}}{\bar{m}^{5 / 2} \bar{K}\left(Z_{i}\right)}\right)^{1 / 4} \\
= & \frac{\pi^{7 / 8} \mathrm{c}}{5^{1 / 4} 2^{7 / 8}}\left(\frac{m_{e}}{\bar{m}}\right)^{5 / 8}\left(\frac{r_{o}}{n_{\mathrm{cr}} \lambda^{4}} \frac{Z_{i} \ln A}{\epsilon \delta_{T}}\right)^{1 / 4} \\
\approx & 0.936 \times 10^{7} \frac{\mathrm{cm}}{\sec }\left(\frac{2 Z_{i}}{A_{i}}\right)^{5 / 8}\left(\frac{1.06 \mu \mathrm{m}}{\lambda}\right)^{1 / 2} \\
& \times\left(\frac{r_{a}}{100 \mu \mathrm{m}} \frac{Z_{i} \ln A}{100 \epsilon \delta_{T}}\right)^{1 / 4} ;
\end{aligned}
$$

$\ln A, \epsilon, \delta_{r}, m_{e}$, and $c$ have the usual meaning.

The continuum model fails above some $\hat{W}$ because the ratio of mean free path to hydrodynamic scale ceases to be small somewhere in the corona, making the classical formula for the heat-flux, $q=-\bar{K} T^{s / 2} \times d T / d r$, inaccurate. A crude recipe for this failure, ${ }^{3,4}$ in order to saturate the flux $\left(|q|<f n T^{3 / 2} m_{e}^{-1 / 2}\right)^{7}$ calls for a difficult choice of the factor $f$. For slab experiments, simulation ${ }^{7}$ and analytical ${ }^{8}$ results have suggested $f \simeq 0.03$ but controversy on this point persists. ${ }^{9}$ In addition, the value of $f$ to use might change with the geometry; taking it independent of position is an overall sim- plification. (This guess is confirmed in pellet experiments by Goldsack et al. ${ }^{10} ; f=0.03$ simulations disagreed with the data, but agreement was attained with $f=0.1$.)

Actually most overall results for pellets are only "piecewise" sensitive to the value of the saturation factor." In particular, the relation between mass ablation rate $\dot{m}$ and ablation pressure $P_{a}$ is independent of $f$ for $f>0.05$ roughly; $P_{a}(W)$ is nearly independent of $f$ for $f>0.08$ and $\hat{W}<10^{4}$. The relation between the critical radius $r_{\mathrm{cr}}$ and $W$ is only weakly dependent on $f^{11,12}$ These results are in accordance with the findings in Ref. 10, where agreement between data and simulations was attained for all $f>0.1$. In these experiments, $5<\hat{W}<5 \times 10^{3}$.

Thus if $f$ is not too low, the classical heat flux (formally corresponding to the limit $f \rightarrow \infty$ ) may be used to calculate overall relations. The exception ${ }^{13}$ is $P_{a}(W)$ at $\hat{W}>10^{4}$. To understand why this relation is sensitive to the value of $f$, note that if saturation is ignored (a) the enthalpy and kineticenergy fluxes are continuous at $r_{c r}$, while $d T / d r$ is not, so that $W / 4 \pi r_{\mathrm{cr}}^{2}=g\left(r_{\mathrm{cr}}^{+}\right)-q\left(r_{\mathrm{cr}}^{-}\right) ;$and $(\mathrm{b})$ if $\hat{W}$ is well above $10^{4}$ (or $r_{\mathrm{cr}} / r_{a}$ above 3) roughly, we have $g\left(r_{\mathrm{cr}}^{+}\right) \simeq W / 4 \pi r_{\mathrm{cr}}^{2}$ $>\left|q\left(r_{c r}^{-}\right)\right| .^{2}$ Saturation then mainly affects the huge outward heat loss, increasing $4 \pi r_{\mathrm{cr}}^{2}\left|q\left(r_{\mathrm{cr}}^{-}\right)\right| / W$ and thus $P_{\mathrm{a}}$, to an extent sensitive to the value of $f$ f for $\hat{W} \sim 10^{7}, P$ shows a three-

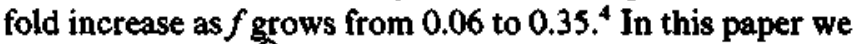
explore this high $W$ regime with a model that makes no appeal to flux saturation. The model is based on the appearance of suprathermal electrons; crucial to the determination of $P_{a}(W)$ is the magnitude of the outward (not the inward) energy transport by these electrons. At the end we compare our results with results from a one-temperature, saturated-flux model.

\section{PHYSICAL MODEL}

A basic analysis ought to include a kinetic evaluation of $q$ under weakly collisional conditions. We look, however, for a simple description, and bypass this current, important problem. The fluid model is modified to allow for two convenient facts:

(i) For $f \geqslant 0.1$, saturation starts in the underdense region, at $\widehat{W} \simeq 10^{3}$ (Fig, 9, Ref. 4); overdense saturation starts at much higher values $\left(\sim 10^{7}\right.$ for $\left.f \simeq 0.3\right)$. For the intermediate regime, evaluation of $q$ is a problem only for $r>r_{c r}$; for 
$r<r_{\text {cr }}$, collisions are frequent enough to warrant a continuum description. This fact is tied to the result $q\left(r_{\mathrm{cr}}^{+}\right)>\left|q\left(r_{\mathrm{cr}}^{-}\right)\right|$ (Sec. I).

(ii) For high intensities, resonant absorption generates suprathermal or hot electrons of temperature $T_{H}>T_{C}$ $\equiv T\left(r_{\mathrm{cr}}\right), T$ being now the main (cold) temperature. ${ }^{14-16}$ For $r>r_{\mathrm{cr}}$, hot electron collisions may be neglected. A large ambipolar field will build up to preserve quasineutrality.

The main consequence of these facts is that the overdense coronal flow is controlled by collision-dominated cold electrons and the underdense flow by collisionless hot electrons.

\section{A. Overdense corona}

Hot electrons entering this region are assumed to contribute negligibly to macroscopic quantities. This assumption is checked at the end. Cold electrons are treated as a continuum fluid with heat flux $-\bar{K} T^{5 / 2} d T / d r$. The equations for the ion-electron fluid are ${ }^{1,2}$

$$
\begin{aligned}
& r^{2} Z_{i} n_{i} v=\mu=\dot{m} / 4 \pi \bar{m} \quad\left(Z_{i} n_{i} \simeq n\right), \\
& \bar{m} n v \frac{d v}{d r}=-\frac{d(n T)}{d r}, \\
& n v\left(\frac{1}{2} \bar{m} v^{2}+\frac{5}{2} T\right)-\bar{K} T^{5 / 2} \frac{d T}{d r}=0 .
\end{aligned}
$$

Both $T$ and $q$ vanish at the cold ablation radius $r_{\sigma}$. We neglect electron inertia, assume spherical symmetry, and take $Z_{i}$ large in order to uncouple the ion temperature (the only quantity sensitive to the charge number). ${ }^{4}$

Let $r_{a}, n_{\mathrm{cr}}, Z_{i}, \bar{m}$, and $P_{a}$, though not $W$, be given. Choosing a value for $\dot{m}$ or $\mu$, Eqs. (2)-(4) can be integrated from $r_{a}$ outward to obtain flow profiles; $\dot{m}$ is then determined by requiring the solution to be univalued at the sonic point. Equation $n\left(r_{\mathrm{cr}}\right)=n_{\mathrm{cr}}$ yields $r_{\mathrm{cr}}$ and both $T_{\mathcal{C}}$ and $v_{\mathrm{cr}}$ $\equiv v\left(r_{\text {cr }}\right)$.

Evaluating (4) at $r_{\mathrm{cr}}^{-}$, and using $\bar{K} T^{5 / 2} \simeq 17.02 \epsilon \delta_{T} l_{e i} n\left(T / m_{e}\right)^{1 / 2}$, where $l_{e i}$ is the ion-electron mean free path, yields

$$
\frac{l_{e i}}{r_{c T}}=\frac{5}{2}\left(\frac{m_{e}}{\bar{m}}\right)^{1 / 2} M_{C}\left(1+\frac{M_{C}^{2}}{5}\right) \frac{d \ln r / d \ln T}{17.02 \epsilon \delta_{r}},
$$

where $M_{c}^{2}=\bar{m} v_{\mathrm{cr}}^{2} / T_{c} ;$ for an Al target,

$$
\frac{l_{e i}}{r_{\mathrm{cr}}} \simeq 7.9 \times 10^{-3} M_{C}\left(1+M_{C}^{2} / 5\right) \frac{d \ln r}{d \ln T} .
$$

Later we will consider $4<\eta_{\mathrm{cr}}<9$; then $0.19>d \ln T /$ $d \ln r>0.08,5<M_{C}^{2}<8$ (see Fig. 2 of Ref. 2), and $0.19<l_{e i} / r_{\text {cr }}<0.72$. Thus the plasma is barely collisional at $r_{\mathrm{er}}$ (of course, $l_{e i} / r$ decreases rapidly with decreasing $r$ ); the fact, however, that $d \ln T / d \ln r<1$ at $r_{\mathrm{cr}}^{-}$makes the heatflux classical for $r<r_{\text {cr }}$, if $f$ is not too low.

\section{B. Underdense corona}

Next, consider $r>r_{\mathrm{cr}}$. The energy equation now reads

$$
4 \pi r^{2}\left(n v \frac{1}{2} \bar{m} v^{2}+n v \leqslant T+q\right)=W .
$$

For low powers, $q=-\bar{K} T^{5 / 2} d T / d r$, and Eqs. (2), (3), and (6), with boundary condition $T(r \rightarrow \infty) \rightarrow 0$, would yield $W .^{2}$ For $\hat{W}$ above $10^{4}$, however, saturation sets in at $r_{\text {cr }}^{+}$for all $f$; evaluating (6) at $r_{\mathrm{ct}}^{+}$with $q=f n T^{3 / 2} / m_{\varepsilon}^{1 / 2}$ yields $W:^{4}$

$$
\dot{m}\left[\frac{1}{2} v_{\mathrm{cr}}^{2}+\frac{T_{c}}{2 \bar{m}}\left(5+f \frac{2 T_{C}^{1 / 2}}{m_{e}^{1 / 2} v_{\mathrm{cr}}}\right)\right]=W .
$$

Unfortunately, when values $0.03<f<0.6$ are allowed for, the indeterminacy in the relation $W\left(P_{a}\right)$ is too large, as shown in Sec. I [the factor $\left(4 T_{c} / m_{e} v_{\mathrm{cr}}^{2}\right)^{1 / 2}$ is large]. form

In this paper we use the energy equation at $r_{\mathrm{cr}}^{+}$in the

$$
\dot{m}\left[\frac{1}{2} v_{\mathrm{cr}}^{2}+\left(T_{H} / 2 \bar{m}\right)(5+\beta)\right]=W,
$$

where $\beta$ is a factor of order unity. This amounts to writing the heat fiux as

$$
q=n v(\beta / 2) T
$$

and choosing $T_{H}$ from between the two temperatures of our model for both enthalpy and heat fluxes. Equation (7b) closes the problem if $T_{H}$ is known.

Equation (8) has been used both in astrophysical and laser-fusion applications. The electron distribution function $F(u, r, t)$ is given by

$$
\left(\mathbf{u} \cdot \nabla+\frac{e}{m_{e}} \nabla \psi \cdot \frac{\partial}{\partial \mathrm{u}}\right) F=\frac{\delta F}{\delta t}
$$

which is a steady Vlasov equation if time variations and collisions, represented by $\delta F / \delta t$, are neglected. Unsteady effects are weak because $r_{o}$ and $\boldsymbol{W}$ change slowly (Sec. I). Collisional effects are stronger for cold electrons. They will be small if $|e \nabla \psi| F /\left(m_{e} T_{c}\right)^{1 / 2}>\left(T_{c} / m_{e}\right)^{1 / 2} F / l_{e} ;$ this condition becomes $T_{H} / T_{C}>r_{\mathrm{cr}} / l_{\mathrm{ei}} \sim 2$. In order to preserve quasineutrality in the presence of hot electrons we must have $|e \nabla \psi| \sim T_{H} / r_{\text {cr }}$.

The steady Vlasov equation clearly yields zero odd moments for $F$ (current, enthalpy flux, heat flux) ${ }^{17.18}$ Corrections caused by $\delta F / \delta t$ will then give small odd moments of same order. ${ }^{17}$ Now, the electron flux at $r_{\text {cr }}{ }^{+}$is known from the conditions of zero total current: $\int F u_{r} d \mathrm{du}=n_{\mathrm{cr}} v_{\mathrm{cr}}$, where $u_{r}$ is the radial component of $u$. Further, since $|e \nabla \psi|>T_{c} / r_{\mathrm{cr}}$, odd moments of $F$, caused by electrons leaking out to infinity, will arise from the hot population. Then, we have at $r_{\mathrm{cr}}^{+}$ the enthalpy flux $\simeq n_{\mathrm{cr}} v_{\mathrm{cr}} \frac{3}{2} T_{H}$ and the heat flux $\simeq n_{\mathrm{cr}} v_{\mathrm{cr}}(\beta / 2) T_{H},{ }^{17}$ leading to $(7 \mathrm{~b})$.

\section{Hot electron temperature}

We take $T_{H}$ from simulations ${ }^{14,15}$ that give

$$
T_{H} \simeq A T_{C}^{\gamma}(1 \mathrm{keV})^{1-\gamma}\left(I_{\mathrm{cr}} \lambda^{2} / 10^{15} \mathrm{~W} \mathrm{~cm}^{-2} \mu \mathrm{m}^{2}\right)^{a},
$$

where $\gamma=1, \alpha=\frac{1}{3}, A=6.5,{ }^{14}$ or $\gamma=0.25, \alpha=0.39$, $A=8.7$ (Ref. 15). Here $I_{\text {cr }}=W / 4 \pi r_{s t}^{2} \phi$ and $\phi$ is the absorption fraction. Equation (10) must be fitted into a basic form. If $E$ and $L$ are the field and length of the resonant plasma wave, then $T_{H} \sim e E L$. Here, $L / \lambda$ is a function of $m_{e} c^{2} / T_{C}$ and $I_{\mathrm{cr}} / c n_{\mathrm{cr}} T_{c}$ (Ref. 14); we also have $E^{2} \propto I_{\mathrm{cr}} / c(\operatorname{Ref}$. 14), or $E^{2} \propto I_{\mathrm{cr}} /\left(T_{H} / m_{e}\right)^{1 / 2} \cdot{ }^{16}$ For a restricted parameter range we may then write

$$
T_{H} \simeq B T_{C}^{\gamma}\left(I_{\mathrm{cr}} / c n_{\mathrm{cr}}\right)^{\alpha}\left(m_{\mathrm{e}} c^{2}\right)^{1-\gamma-\alpha} .
$$

Comparing (10) and (1 la) we obtain $T_{H} \propto c^{1 / 3}$ for both $\gamma=\frac{1}{3}$ and $\gamma=0.25$. Accordingly we set $\alpha=(5-6 \gamma) / 9$. 
We rewrite (11a) in the form

$T_{H} \simeq B\left(\frac{m_{\varepsilon}}{\bar{m}}\right)^{(1-3 \gamma) / 9} \frac{T_{c}^{\gamma}\left(\bar{m} V^{2}\right)^{1-\gamma}}{\left(\bar{m} V / m_{e} c\right)^{1 / 3}}\left(\frac{\hat{W}}{4 \pi \phi} \frac{r_{a}^{2}}{r_{\mathrm{sr}}^{2}}\right)^{(5-6 \gamma / / 9}$.

Equation (11b) shows that resonant absorption effects are parametrized by the dimensionless number $\widehat{V} \equiv \bar{m} V / m_{e} c$. (Inverse bremsstrahlung effects are parametrized by $\hat{V}$, too. $\left.{ }^{12}\right)$ We use (11b) in (7b). To make (11b) agree with (10), to within $1 \%$ for both $\gamma=\frac{1}{3}$ and $\gamma=0.25$, we set $\left.B\left(\bar{m} / m_{e}\right)^{\prime \prime-3}\right) / 9=1.35$.

\section{OVERDENSE HOT EFFECTS}

We now justify neglecting hot electron effects in the overdense region. For simplicity, we estimate them assuming that ingoing hot electrons at $r_{\mathrm{cr}}$ are isotropic and monoenergetic. They scatter off high $-Z_{i}$ ions at a rate determined by the $90^{4}$-defiection time $t_{D}=m_{e}^{2} w^{3} / 8 \pi Z_{i} n e^{4} \ln A,{ }^{3}$ and lose energy to cold electrons at a slower rate of characteristic time $Z_{i} t_{D} ; E_{H} \equiv m_{e} w^{2}$ is the hot energy. Then

$$
\begin{aligned}
& \frac{d\left\langle s^{2}\right\rangle}{d t}=w^{2} t_{D}=\frac{2 E_{H} t_{D}}{m_{e}}, \\
& \frac{d E_{H}}{d t}=\frac{-E_{H}}{Z_{i} t_{D}},
\end{aligned}
$$

where $\left\langle s^{2}\right\rangle$, the mean squared distance from $r_{c r}$, obeys a random-walk law. We assume that hot electrons remain "marked hot" as they lose energy (see Ref. 3 for an alternative description). Then we have

$$
\text { hot density }=n_{H}(r) \simeq n_{H}\left(r_{\mathrm{cr}}\right) .
$$

For electrons moving radially on the average we set $\left\langle s^{2}\right\rangle=\left(r_{\text {cr }}-r\right)^{2}$. From $(12)$ and $(13)$, we obtain

$$
\frac{d E_{H}}{d r}=\frac{m_{e}\left(r_{\mathrm{cr}}-r\right)}{Z_{l} t_{D}^{2}}
$$

The largest hot electron effects on the ion-plus-coldelectron fluid should be caused by energy exchange. Hence, we neglect hot density, current, and pressure, against the corresponding cold quantities. We neglect $3 T / 2$ against $E_{H}$ also. Consequently (4) becomes

$$
\frac{1}{r^{2}} \frac{d}{d r} r^{2}\left[n v\left(\frac{1}{2} \bar{m} v^{2}+\frac{5}{2} T\right)-\bar{K} T^{5 / 2} \frac{d T}{d r}\right]=\frac{n_{H} E_{H}}{Z_{i} t_{D}} ;
$$

Eqs. (2) and (3) are not modified. The analysis of the overdense region is then given by Eqs. (2)-(4) for $r_{a}^{*}<r<r_{\mathrm{th}}$, and by (2), (3), (16), (14), and (15) for $r_{\text {th }}<r<r_{\mathrm{cr}}$. The boundary condition on (15) is $E_{H}\left(r_{\mathrm{cr}}\right)=3 T_{H} / 2 ; r_{\mathrm{th}}$ is determined by a thermalization condition, $E_{H}\left(r_{\mathrm{th}}\right)=3 T\left(r_{\mathrm{th}}\right) / 2$.

We carried out calculations for different values of $n_{H}\left(r_{\mathrm{cr}}\right) / n_{\mathrm{cr}}$. For low values we obtain the cold $n, v, T$ profiles, as well as $r_{\mathrm{th}}$, and $E_{H}$ for $r_{\mathrm{th}}<r<r_{\mathrm{cr}}$. As $n_{H}\left(r_{\mathrm{sr}}\right) / n_{\mathrm{cr}}$ increases the profiles change and the power going into the cold electrons $\left(4 \pi r_{\mathrm{cr}}^{2} \times \bar{K} T_{c}^{s / 2} d T /\left.d r\right|_{r_{\mathrm{cr}}^{-}}\right)$decreases. This power vanishes at about $n_{H}\left(r_{\mathrm{cr}}\right) / n_{\mathrm{cr}}=\mathrm{f}$. At this point, the overall relations $r_{\mathrm{cr}}(W)$ and $P_{s}(W)$ differ by a few percent from results that ignore hot-energy deposition, even though the ion- plus-cold-electron flow is sensibly modified. We conclude that overdense hot effects may be neglected for all reasonable values of $n_{H}\left(r_{\mathrm{cr}}\right) / n_{\mathrm{cr}}$, which is a free parameter of the analysis. We found $r_{\text {th }}>r_{0}$ always.

\section{DISCUSSION}

Figure 1(a) and (b) shows $r_{\mathrm{cr}} / r_{a}$ and $P_{c} / \rho_{\mathrm{cr}} V^{2} \mathrm{vs} \hat{W}$ for a few values of $\hat{\boldsymbol{V}}$. Numerically

$$
\begin{aligned}
& \hat{W} \simeq \frac{0.682 W}{10^{9} \text { watts }}\left(\frac{A_{i}}{2 Z_{i}}\right)^{7 / 8}\left(\frac{\lambda}{1.06 \mu \mathrm{m}}\right)^{7 / 2} \\
& \times\left(\frac{100 \mu \mathrm{m}}{r_{a}}\right)^{11 / 4}\left(\frac{100 \epsilon \delta_{T}}{Z_{i} \ln A}\right)^{3 / 4}, \\
& \hat{V} \simeq 1.14\left(\frac{A_{i}}{2 Z_{i}}\right)^{3 / 8}\left(\frac{1.06 \mu \mathrm{m}}{\lambda}\right)^{1 / 2}\left(\frac{r_{a}}{100 \mu \mathrm{m}} \frac{Z_{i} \ln A}{100 \epsilon \delta_{T}}\right)^{1 / 4} .
\end{aligned}
$$

Results from Ref. 4 for several values of $f$ are also shown for comparison. ${ }^{19}$

Both the present and the saturated-flux, one-temperature, models are simple approximations. For the second one,
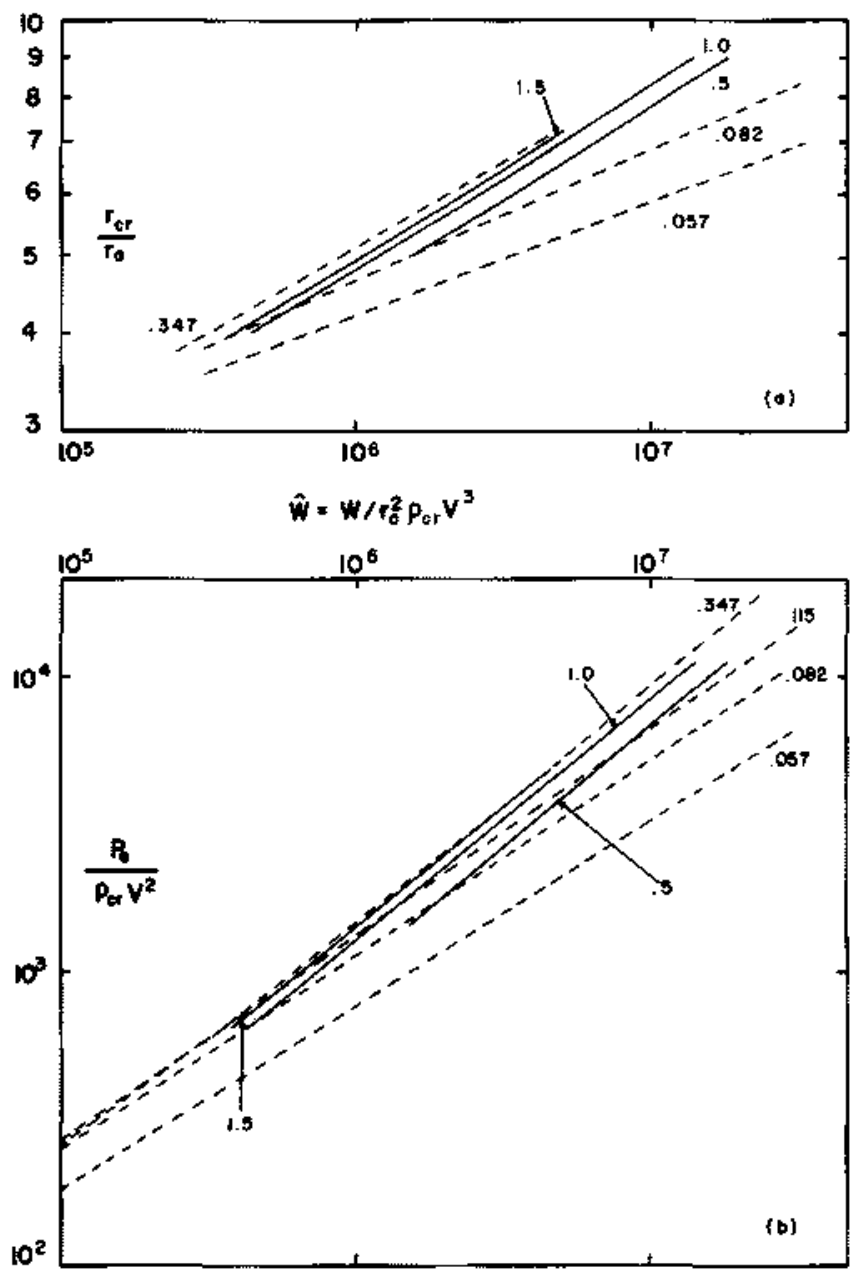

FIG. 1. Critical radius $t_{c}(a)$ and ablation pressure $P_{a}(b)$ versus absorbed laser power $W$, as found here ( $(-)$ and in Ref, $4(\cdots)$ for a few values of $\hat{V}$ and $f\left(A_{i} / 2 Z_{i}\right)^{1 / 2}$, respectively. Here, $V, \hat{W}$, and $\hat{V}$ are defined in Eqs. (1), (17), and $(18) ; f$ is the flux saturation factor, $r_{\sigma}$ is the ablation radius, $\rho_{\mathrm{cr}}$ is the mass critical density, and $A_{i}$ and $Z_{i}$ are the ion mass and charge number, respectively. 
however, we still have to choose a value for $f$; this is certainly a questionable matter for pellets and makes results at high $\hat{W}$ useless. Figure $1(\mathrm{~b})$ shows that for $\hat{W}=10^{7}, P_{a}$ increases by a factor of 3 in going from $f \simeq 0.06$ to $f \simeq 0.35\left[r_{\text {cr }}\right.$ in Fig. $1(a)$ increases by a factor of 1.5 ]; when values $f=0.03$ and 0.6 are considered ${ }^{3,4}$ the factor is larger than 10 (a 1000\% indeterminacy). In the present model $\hat{V}$ is a known parameter. The indeterminacy arises from $10 \%$ errors in the approximations, and from the factor $(1+\beta / 5) \phi^{(6 y-5) / 9}$, after using (11b) in (7b). To draw Fig. 1 we set $\beta=0, \phi=0.4$, and checked that $P_{o}$ and $r_{\mathrm{cr}}$ changed by $15 \%$ and $5 \%$, respectively, for $\beta= \pm 1$; for $\phi=0.5$ or 0.3 , changes were half as large. There is no reason to expect $\beta$ to be large. Thus, results should be accurate to within $50 \%$ at worst. From Fig. 1 we may conclude that the saturated-flux model with $f=0.03$ or 0.6 does not agree with the present model; agreement requires setting $f=0.1$ to 0.3 .

Our analysis requires a profile steepening at $r_{\mathrm{cr}}$ that is neither too weak (resonant absorption and electron acceleration must be significant) nor too strong (the upper density in the step profile must be close to $n_{\mathrm{cr}}$, as is implicitly assumed in the model). This was ensured by making $I_{\mathrm{cr}} \lambda^{2}$ lie between the lowest values studied in Refs. 15 and $14\left(0.3\right.$ and $5 \times 10^{15}$ $\mathrm{W} \mathrm{cm}{ }^{-2} \mu \mathrm{m}^{2}$, respectively); in all cases $I_{\mathrm{cr}} / \mathrm{cn}_{\mathrm{cr}} T_{c}$ lay between its lowest values in Ref. $15(0.05)$ and Ref. $14(0.4)$ [accordingly we set $\gamma=0.25$ in (11b)]. The range of validity of present results starts at about $\hat{W} \simeq 3 \times 10^{5}$ and reaches above $10^{7}$; it extends significantly the results from Refs. 2, 4, and 12 , which extended up to about $3 \times 10^{4}$.

The range depends on $\hat{V}$ as is roughly shown in Fig. 1 ; it narrows at both low and high $\hat{V}$. This is because $\hat{W} \propto I_{\mathrm{cr}} \lambda^{2} \hat{V}^{-3}$. At low $\hat{V}$, and for $I_{\mathrm{cr}} \lambda^{2}$ at its upper bound, $\hat{W}$ (or $r_{\mathrm{cr}} / r_{a}$ ) is too large; $l_{e l} / r_{\mathrm{cr}}$ exceeds unity and Spitzer's flux fails at $r_{\mathrm{cr}}^{-}$. At high $\hat{V}$ and $I_{\mathrm{cr}} \lambda^{2}$ at its lowest bound, $r_{\mathrm{cf}} / r_{\mathrm{a}}$ is too low: hot electrons thermalize at $r_{\mathrm{cr}}$ $\left(r_{t h} \simeq r_{\text {er }}\right)$. Fortunately $\hat{V}$ is close to unity for most experiments of interest.

\section{ACKNOWLEDGMENT}

This research was sponsored by the Comisión Asesora de Investigación Científico-Técnica of Spain.

'Yu. V. Afanas'ev, E. G. Gamalii, O. N. Krokhin, and V. B. Rozanov, Sov. Phys. JETP 44, 311 (1977); S. J. Gitomer, R. L. Morse, and B. S. Newberger, Phys. Fluids 20, 234 (1977).

${ }^{2} J$. Sanz, A. Lirián, M. Rodriguez, and J. R. Sanmartín, Phys. Fluids 24, 2098 (1981)

${ }^{3}$ C. E. Max, C. F. Mckee, and W. C. Mead, Phys. Fluids 23, 1620 (1980).

${ }^{4} \mathrm{~J}$. Sanz and J. R. Sanmartín, Phys. Fluids 26, 3361 (1983).

SL. Spitzer, Physics of Fully Ionised Gases (Wiley, New York, 1967), Chap. 5.

'In Eq. (20) of Ref. 4, defining $V$, the exponent i was missing from $\bar{m}$. Here temperature $T$ is messured in energy units.

${ }^{7}$ W. L. Kruer, Comments Plasma Phys. 5, 69 (1979).

${ }^{8} R$. Ramis and J. R. Sanmartin, Nucl. Fusion 23, 739 (1983).

${ }^{9}$ N. St. J. Braithwaite, A. Montes, and L. M. Wickens, Plasma Phys. 23, 713 (1981).

10T. J. Goldsack, J. D. Kilkenny, B. J. MacGowan, P. F. Cunningham, C. S. Lewis, M. H. Key, and P. T. Rumsby, Phys. Fluids 25, 1634 (1982).

'tSee Figs. 10 and 11 of Ref. 4.

${ }^{12}$ In Refs. 1-4 absorption was assumed to 0 cur at $n_{\mathrm{cr}}$. Inverse bremsstrahlung absorption at $n<n_{\mathrm{er}}$ has been studied by J. Nicoliss and J. R. Sanmartín, submitted to Plasma Phys. and Controlled Fusion.

${ }^{13}$ Since $\dot{m}\left(P_{a}\right)$ is independent of $f, \dot{m}(W)$ will depend on $f, t o 0$.

${ }^{14}$ D. W. Forslund, J. M. Kindel, and K. Lee, Phys. Rev. Lett. 39, 284 (1977).

${ }^{15}$ K. Estabrook and W. L. Kruer, Phys. Rev, Lett. 40, 42 (1978).

16J. R. Albritton and A. B. Langdon, Phys. Rev. Lett. 45, 1794 (1980).

1'E. J. Valeo and I. B. Bernstein. Phys, Fluids 19, 1348 (1976).

${ }^{18} \mathrm{P}$. Mora and R. Pellat, Phys. Fluids 22, 2300 (1979).

19 The relations between $r_{\mathrm{er}} / r_{a}$ and $P_{a} / \rho_{\mathrm{cs}} V^{2}$, and $r_{\mathrm{cr}} / r_{\mathrm{a}}$ and $\dot{m} / r_{\mathrm{s}}^{2} \rho_{\mathrm{cr}} V$ are the same as in Ref. 2, because the overdense analysis is the same. 
